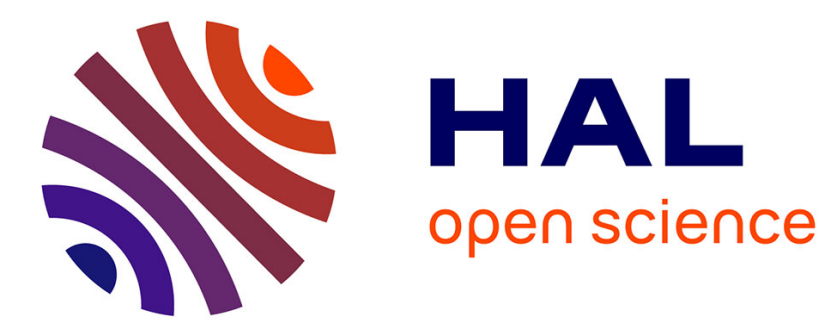

\title{
Slowing Quantum Decoherence by Squeezing in Phase Space
}

H Le Jeannic, A Cavaillès, K Huang, R Filip, J Laurat

\section{To cite this version:}

H Le Jeannic, A Cavaillès, K Huang, R Filip, J Laurat. Slowing Quantum Decoherence by Squeezing in Phase Space. Physical Review Letters, 2018, 120, 10.1103/physrevlett.120.073603 . hal-03269647

\section{HAL Id: hal-03269647 https://hal.science/hal-03269647}

Submitted on 24 Jun 2021

HAL is a multi-disciplinary open access archive for the deposit and dissemination of scientific research documents, whether they are published or not. The documents may come from teaching and research institutions in France or abroad, or from public or private research centers.
L'archive ouverte pluridisciplinaire HAL, est destinée au dépôt et à la diffusion de documents scientifiques de niveau recherche, publiés ou non, émanant des établissements d'enseignement et de recherche français ou étrangers, des laboratoires publics ou privés. 


\title{
Slowing Quantum Decoherence by Squeezing in Phase Space
}

\author{
H. Le Jeannic, ${ }^{1}$ A. Cavaillès, ${ }^{1}$ K. Huang, ${ }^{1,2}$ R. Filip, ${ }^{3, *}$ and J. Laurat ${ }^{1, \dagger}$ \\ ${ }^{1}$ Laboratoire Kastler Brossel, Sorbonne Université, CNRS, PSL Research University, Collège de France, \\ 4 Place Jussieu, 75005 Paris, France \\ ${ }^{2}$ Shanghai Key Laboratory of Modern Optical Systems, and Engineering Research Center of Optical Instruments \\ and Systems (Ministry of Education), School of Optical Electrical and Computer Engineering, \\ University of Shanghai for Science and Technology, Shanghai 200093, China \\ ${ }^{3}$ Department of Optics, Palacký University, 17. listopadu 1192/12, 77146 Olomouc, Czech Republic
}

(Received 19 July 2017; revised manuscript received 31 October 2017; published 13 February 2018)

\begin{abstract}
Non-Gaussian states, and specifically the paradigmatic cat state, are well known to be very sensitive to losses. When propagating through damping channels, these states quickly lose their nonclassical features and the associated negative oscillations of their Wigner function. However, by squeezing the superposition states, the decoherence process can be qualitatively changed and substantially slowed down. Here, as a first example, we experimentally observe the reduced decoherence of squeezed optical coherent-state superpositions through a lossy channel. To quantify the robustness of states, we introduce a combination of a decaying value and a rate of decay of the Wigner function negativity. This work, which uses squeezing as an ancillary Gaussian resource, opens new possibilities to protect and manipulate quantum superpositions in phase space.
\end{abstract}

DOI: 10.1103/PhysRevLett.120.073603

The coupling of a quantum system to an ambient environment leads to the so-called decoherence phenomenon [1]. This irreversible process washes out the nonclassical features of quantum states and constitutes a main impediment to quantum information sciences [2], including for quantum computing, metrology, and communication. In quantum physics, decoherence typically scales with time, strength of the coupling, and also with an effective dimension of the system. Larger systems exhibit faster decoherence and are extremely fragile.

A paradigmatic example is the superposition of coherent states (CSS), also known as Schrödinger cat state. This state of the form $|\alpha\rangle \pm|-\alpha\rangle$ consists of a superposition of coherent states with opposite phases and a mean number of energy quanta $|\alpha|^{2}$. In the recent years, CSSs have been prepared in a variety of experimental platforms, including trapped-ion setups [3-7], photonics experiments [8-13], cavity-QED systems [14], and superconducting circuits [15]. In subsequent studies, these states have been used to follow their decoherence under energy loss and to explore the boundary between the classical and quantum worlds $[4,14,16]$.

In this context, over the past several years, different strategies have been developed to mitigate the effect of decoherence and protect nonclassical features. Errorcorrecting codes $[17,18]$, protected logical qubits with redundant information encoding [19], or probabilistic quantum distillation protocols [20] are actively pursued. Physical control of decoherence via measurement and feedback has also been proposed and implemented $[21,22]$. In all these approaches, the effect of decoherence on a given state is alleviated afterwards, without an engineering of the environment. By a challenging quantum squeezing of the environmental bath, the decoherence can be reduced as well $[23,24]$.

In contrast, another equivalent but potentially more feasible strategy can consist of slowing the decoherence by acting on the state itself. Such protection yet to be demonstrated can be obtained for instance via a Gaussian squeezing operation that adapts the non-Gaussian state prior to the channel [24-26]. The squeezing process does not preserve energy and translates in phase space to a simple scale transformation that compresses one direction and dilates the orthogonal one. By definition, it therefore preserves the non-Gaussian content of the initial state, and in particular the negative fringes in the Wigner function representation in phase space [27]. Squeezing constitutes the essential and broadly available resource in continuousvariable quantum optics but recent proposals have also shown the usefulness of Gaussian operations on nonGaussian states [28]. Moreover, a deterministic squeezing gate has been recently demonstrated and is now available for optical protocols [29].

In this Letter, we report first quantitative measurements that demonstrate a decoherence much slower than the standard one typically observed for large coherent-state superpositions. Specifically, we experimentally generate a squeezed optical version of CSS and follow the behavior of the Wigner function oscillations under photon loss. We simultaneously determine the decaying value and a so-called rate of decay of the Wigner function negativity and compare it with standard decoherence. We demonstrate 

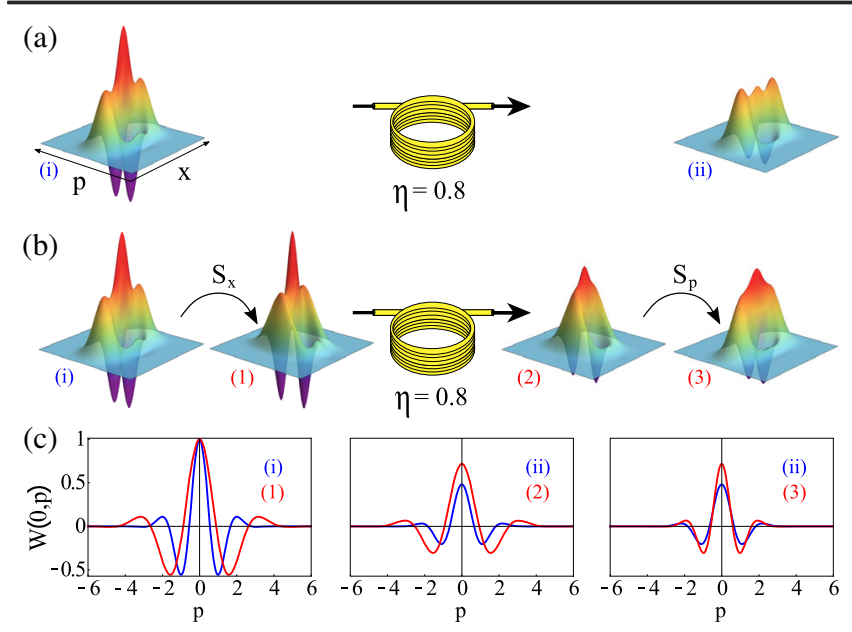

FIG. 1. Illustration of the decoherence process in phase space and protection by a squeezing operation. (a) Direct transmission. The theoretical Wigner functions of an even coherent-state superposition $\left(|\alpha|^{2}=2\right)$ are displayed before and after propagation through a lossy channel with a transmission $\eta=0.8$. (b) Squeezing adaptation before transmission. The same coherent-state superposition is first squeezed $\left(S_{x}=4 \mathrm{~dB}\right)$ and then transmitted. An antisqueezing operation may then be performed. (c) The 2D plots give the cross sections of the Wigner functions along the $p$ axis. The oscillations are damped with the loss but the negativities are better preserved for the squeezed version.

that both are substantially reduced for the squeezed CSS. Such observations therefore elevate the squeezing operation as a fundamental off-line Gaussian resource for quantum information processing.

To first illustrate the Gaussian adaptation strategy, Fig. 1 depicts the specific case considered in this study. A squeezed coherent-state superposition and a CSS with the same amplitude but without squeezing propagate over a lossy channel. The theoretical Wigner functions and their cross sections are given before and after the in-line attenuation. As can be seen, the Wigner function oscillations are damped with the loss but the fringe contrast and the negativities are better preserved for the squeezed superposition. If necessary, depending on the subsequent use, antisqueezing can be applied after propagation. We will not consider this additional operation in the following as it does not change the Wigner negativity values.

Gaussian adaptation.-We begin by considering a lossy Gaussian channel with a transmission $\eta$, and a squeezing operation on the environmental bath with an amplitude gain $\sqrt{G}$ [squeezing $S$ in dB, $S=-10 \log (G)$ ]. After the channel, the noncommuting operators corresponding to the phasespace variables are transformed following the relations

$$
\begin{aligned}
& X_{\text {out }}=\sqrt{\eta} X_{\text {in }}+\sqrt{1-\eta} \sqrt{G} X_{\text {env }}, \\
& P_{\text {out }}=\sqrt{\eta} P_{\text {in }}+\sqrt{1-\eta} \sqrt{G^{-1}} P_{\text {env }} .
\end{aligned}
$$

The uncorrelated operators $X_{\text {env }}$ and $P_{\text {env }}$ stand for the environmental noise and the correlation between them can always be canceled by properly changing the phase before and after the channel. By combining squeezing and phase shift, this noise can be therefore arbitrarily canceled in any direction of the phase space at the cost of an increase in the perpendicular direction. From these expressions, it also follows that the strategy consisting of squeezing the initial state is equivalent to the challenging squeezing of the environmental noise, as suggested in Ref. [24]. We note, however, that in Ref. [24], the decoherence is studied via the evolution of the state purity. Purity as well as fidelity are parameters that average all features of the considered state and do not directly relate to the important nonclassical aspects of the interference in phase space.

Turning to the phase-space representation, the Wigner function $W(x, p)$ of the initial state is thereby transformed to $W^{\prime}\left(x^{\prime}, p^{\prime}\right)$ at the channel output by a two-dimensional convolution with a kernel depending on the environment [27]

$$
\begin{aligned}
& K\left(x, p, x^{\prime}, p^{\prime}\right) \\
& \propto \exp \left(-\frac{\left(x^{\prime}-\sqrt{\eta} x\right)^{2}}{2 G(1-\eta) V_{x, \text { env }}}-\frac{\left(p^{\prime}-\sqrt{\eta} p\right)^{2}}{2 G^{-1}(1-\eta) V_{p, \text { env }}}\right),
\end{aligned}
$$

where $V_{x, \text { env }}$ and $V_{p \text {,env }}$ are variances of the environmental noise. Because of the global nature of the convolution in the phase space, the value and direction of the squeezing has to be optimized separately for different input states $W(x, p)$ and for different measures of decoherence.

Using Eq. (2) for a purely lossy channel, the output Wigner function $W^{\prime}(0,0)$ is clearly non-negative for any $\eta<1$ if the gain $G$ asymptotically tends to zero or diverges to infinity. Simultaneously, for the states with $W(0,0)<0$ and a nonvanishing derivative $d W^{\prime}(0,0) /\left.d G\right|_{G=1}$, a squeezing along the $x$ or $p$ quadratures helps to slow down decoherence. As a result, there exists an optimal squeezing value. The derivative gives a sufficient integral condition to reach it. Moreover, as any negativity of $W(x, p)$ can be shifted to the origin, this analysis applies therefore to many asymmetrical states in phase space. The simplest examples have been theoretically presented in Ref. [26] and optimally squeezed CSSs are derived in the Supplemental Material [30].

Decoherence quantification.-We now turn to the quantification of decoherence. Any modulation of the Wigner function into the highly-nonclassical negative values gradually decays under increasing dissipation to a zerotemperature environment [31]. The presence of such negative values is a clear witness of nonclassical phase space interference. However, it is not sufficient to describe the dynamics of decoherence in the channel.

For this purpose, we adapt the approach initiated by Zurek and co-workers using a relative rate $\dot{W}(x, p) / W(x, p)$, where the dot denotes time derivative in Ref. [31]. Our steps beyond are the following. First, the derivative can be respective to 


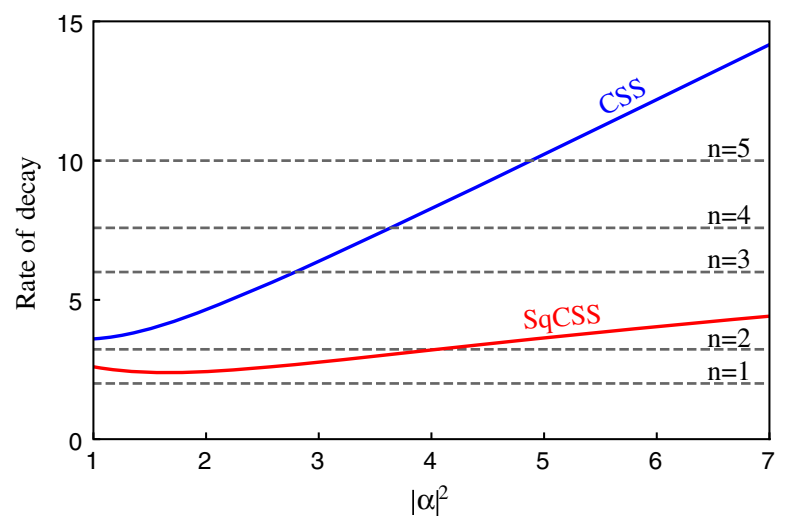

FIG. 2. Protection of coherent-state superpositions by squeezing. The rates of decay at $\eta=1$ for CSS (blue solid line) and optimally squeezed CSS (red solid line) are plotted as a function of the mean photon number $|\alpha|^{2}$. For comparison, the horizontal gray dashed lines give the rate of decay for Fock states $|n\rangle$.

any parametrization of the decay. In our case, it is the channel transmission $\eta$. Second, we focus only on the decay of negative values of the Wigner function. Third, we specifically measure the rate of the decay of the largest negativity located at the coordinate $x_{\min }$ and momentum $p_{\min }$ at the given transmission $\eta$. Therefore, we use the rate of decay defined as

$$
R D_{W_{\eta}}=\left.\frac{1}{W\left(x_{\min }, p_{\min }, \eta\right)} \frac{\partial W\left(x, p, \eta^{\prime}\right)}{\partial \eta^{\prime}}\right|_{x_{\min }, p_{\min }, \eta} .
$$

In Fig. 2, we present the evolution of the rate of decay for a CSS with a size $|\alpha|^{2}$, at a transmission $\eta=1$, as well as the value for several Fock states for comparison. For the Fock states $|1\rangle$ and $|2\rangle$, the rate of decay is equal to $R D_{|1\rangle}=2$ and $R D_{|2\rangle}=3.22$ for $\eta=1$, demonstrating that the decay is faster for the higher Fock states. More generally, for any two Fock states $|n\rangle$ and $|n+1\rangle$, we obtain $R D_{|n+1\rangle}>R D_{|n\rangle}$. For CSSs with different amplitudes, we have as well $R D(\alpha+\Delta \alpha)>R D(\alpha)$. For a large mean photon number, Fock states and CCSs tend to have a comparable rate of decay, which increases with the size of the system (see the Supplemental Material [30]). As illustrated in Fig. 2, squeezing a CSS enables us to strongly reduce the rate of decay and to make it comparable to the one of smaller Fock states. This reduction is even greater for CSS with large size [26].

Experimental generation of squeezed optical CSS.-We proceed to the experimental investigation. We generate freepropagating squeezed even CSSs via our recently demonstrated versatile method based on two-photon heralding [13]. This scheme relies on a two-mode squeezed vacuum state emitted by a type-II optical parametric oscillator (OPO) operated far below threshold [32] and linear mixing of the two entangled modes (see the Supplemental Material [30]). A two-photon detection based on high-efficiency superconducting nanowire single-photon detectors [33] on one of (a)

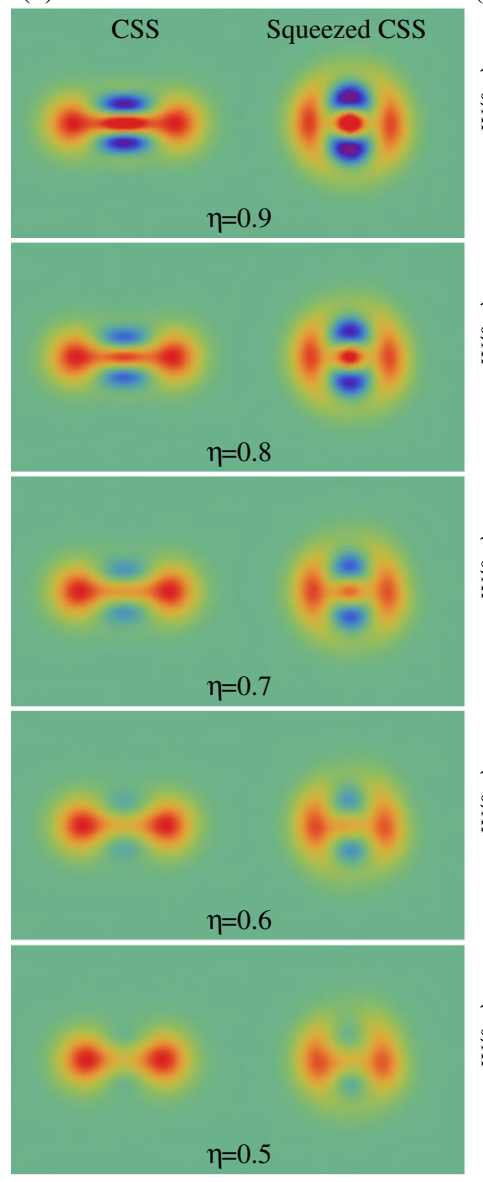

(b)
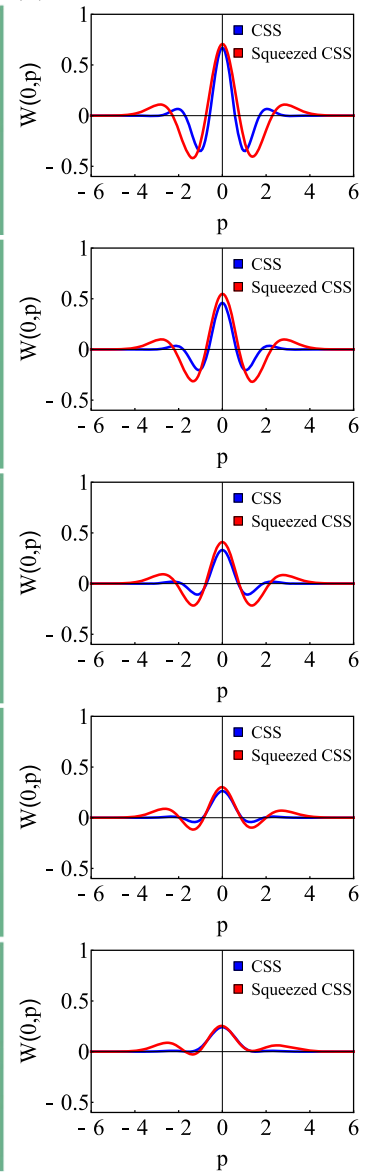

FIG. 3. Experimental results. (a) The Wigner functions of the experimental squeezed optical CSS are given for five different values of channel transmission $\eta$ and compared with a CSS of same amplitude without squeezing. The initial synthesized state exhibits a fidelity $F=77 \%$ with a squeezed CSS with $|\alpha|^{2}=2.1$ and a squeezing value $S=4 \mathrm{~dB}$. (b) The right column provides the cross sections along the imaginary axes. The initial state corresponding to $\eta=0.9$ is corrected for $15 \%$ detection losses. The specific value $\eta=0.9$ corresponds to the estimated escape efficiency of the OPO.

the resulting modes heralds the generation of a superposition of zero and two-photon Fock states on the other. This superposition can be tuned with the adjustable linear mixing and shows large fidelity with the targeted squeezed CSS for $|\alpha|^{2}$ as large as 3. Experimental details have been reported elsewhere [13,34].

The heralded state is characterized by full quantum state tomography via homodyne detection. Quadrature values from 50000 measurements are processed with a maximum likelihood algorithm [35], which provides the density matrix and the associated Wigner function. The Wigner function is given in Fig. 3 (panel $\eta=0.9$ ), after correction for detection losses (15\%). The state exhibits a fidelity $F \sim 77 \%$ with a squeezed CSS with $|\alpha|^{2}=2.1$ and $S=4 \mathrm{~dB}$. The negative peaks of the Wigner functions reach $W=-0.32 \pm 0.01$. 

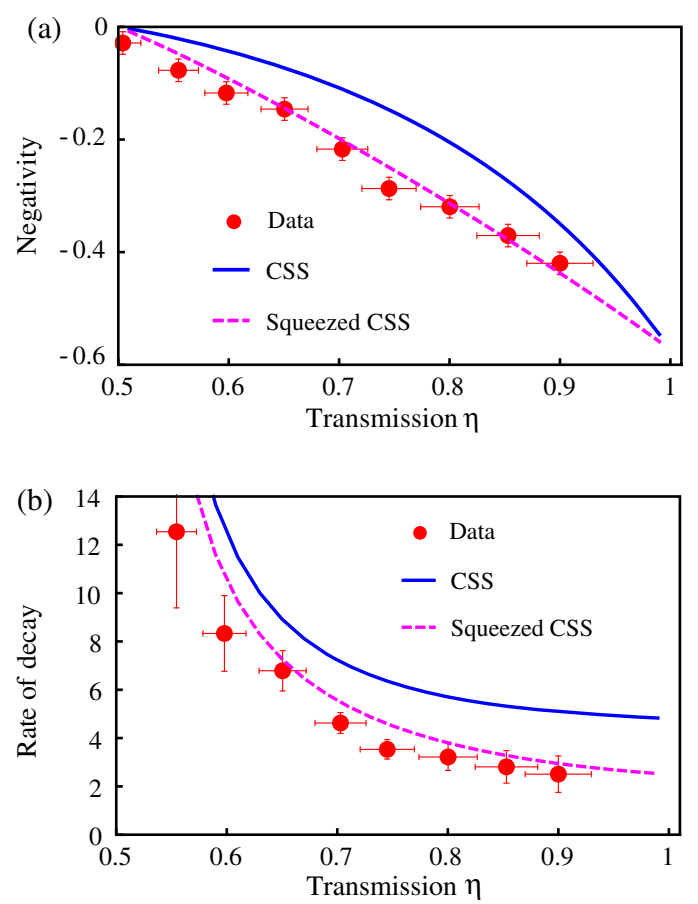

FIG. 4. Evolution of the Wigner function negativity and rate of decay as a function of the transmission $\eta$. (a) Negativity of the Wigner function of the initial squeezed CSS with $|\alpha|^{2}=2.1$ and $S=4 \mathrm{~dB}$. (b) Estimated rate of decay of the negativity. The solid lines show the evolution for the CSS of the same amplitude without squeezing. The dashed lines correspond to the theoretical model.

The aim of the present investigation is to study the effect of losses on this squeezed CSS. For this purpose, we introduce losses by changing the temporal mode used for the homodyne characterization [36,37]. Indeed, because of the continuous-wave nature of our experiment, the state reconstruction requires a temporal filtering. The temporal mode is given by a double-decaying exponential profile $f(t)=\sqrt{\pi \gamma} e^{-\pi \gamma|t|}$, where $\gamma$ is the bandwidth of the OPO cavity. By mismatching the temporal mode and the optimal one, i.e., by adding a delay $\tau$ in the reconstruction algorithm, we introduce controllable loss, with an effective overall transmission $\eta$. This procedure works because the orthogonal modes are very close to the vacuum state as the OPO is pumped far below threshold. It provides the states after the lossy channel and these decohered states are used in the rest of the study.

Experimental decoherence for squeezed optical CSS.The evolution of the Wigner function under decoherence is compared for the experimentally prepared squeezed CSS and for the CSS of the same amplitude but without squeezing. Figure 3(a) shows the Wigner functions for five different values of channel transmission $\eta$ and Fig. 3(b) provides the corresponding cross sections along the imaginary axis. Decoherence manifests itself in the progressive reduction of the Wigner function negativities. This comparison confirms that these negativities are better preserved for the squeezed CSS.

More quantitatively, Fig. 4(a) provides the maximal Wigner function negativity as a function of the transmission $\eta$. We see that the negativity stays larger for the squeezed superposition, whatever the amount of loss experienced by the state. By fitting the experimental points by a third-order polynomial, we can finally estimate the derivative of the negativity, and therefore the rate of decay as a function of the transmission $\eta$, as shown in Fig. 4(b). The protection simultaneously improves both the negativity and the rate of decay, for any losses. For example, for losses around 20\%, the rate-of-decay is typically decreased by about a factor 2 , a very substantial improvement. The pink dashed lines correspond to a simple theoretical model taking into account the mean-photon number and the squeezing value. The small discrepancy is explained by the higher photonnumber components included in this model and not present in our experimentally synthesized superposition.

Discussion.-Our result clearly illustrates the decoherence protection provided by the squeezing adaptation of fragile nonclassical feature in the coherent-state superpositions. This improved robustness can find immediate applications in quantum communication and computing schemes. It has been shown, for instance, that CSS code words can be used for quantum memory protection [38] and this study has been extended to multicomponent superpositions [39]. The use of squeezed code words may reduce further the error rate. In a similar way, recent demonstrations based on CSSs for universal gate implementations in superconducting circuits [40] could benefit from this approach to further limit errors. Protecting nonclassical features in experiments where such states are used to study various topological effects is also highly relevant [41]. The general idea to optimize CSSs can be analyzed for a variety of other catlike states used in different applications proposed sometimes long-time ago, such as entangled CSSs for quantum repeaters [42] or compass states for quantum metrology [43].

Beyond catlike states, this strategy can be applied to protect other quantum states. For lossy channels, it is especially powerful for the phase-asymmetrical states that exhibit complex topology of negativity in phase space $[44,45]$. These local operations may also provide advantages for protecting complex bipartite states, including NOON states or hybrid entangled states [46,47]. However, as stated before, an exact prediction for a specific state must be analyzed always relatively to a given figure of merit. The channel is described by a convolution of the input Wigner function with a kernel representing the channel noise. As a nonlocal transformation in phase space, the convolution forbids for a general state any simple intuitive picture of how and why the squeezing helps. It is not exactly related to minimization of energy or to azimuthal symmetry in phase space. Importantly, the nonlocal nature of this transformation 
means involvement of all small details of the Wigner function in the decoherence process. A general operational procedure is therefore dependent not only on the specific state but also on the feature to protect for a given application.

The squeezing adaptation used here can be further generalized to any Gaussian operation. If we accept a probabilistic protocol, any conditional purity-preserving Gaussian filter can be added to the toolbox to protect against decoherence [48]. For multimode states, useful for example in quantum coding, multimode Gaussian operations and filters can also be advantageous. Beyond the lossy channel, the method is extendable to noisy and nonGaussian channels [49].

In conclusion, we have shown that the photon-loss induced decoherence of CSSs can be significantly slowed down by initially squeezing the superposed states. This strategy allows us here to reduce the rate of decay by a factor of 2 . Theory remarkably predicts even higher factors for larger superpositions. Our study thereby confirms the usefulness of Gaussian operations on non-Gaussian states to make them more robust and to approach a minimal decoherence rate. This Gaussian adaptation is a versatile tool for advanced quantum state engineering and robust state transfer, and can find immediate applications in a variety of schemes. This work also invites to revisit the standard decoherence rate usually considered for many other physical platforms, where quantum superpositions of macroscopic states can be generated. In a broader context, this result constitutes a further example of the potential of the hybrid quantum information processing approach where Gaussian and non-Gaussian operations and techniques can be combined for advanced capabilities and improved scalability [50].

The authors thank O. Morin, Y.-C. Jeong, and J. Ruaudel for their contributions in the early stage of the experiment. This work was supported by the European Research Council (Starting Grant HybridNet). R. F. also acknowledges funding by Grant No. GB14-36681G of the Czech Science Foundation. J. L. is a member of the Institut Universitaire de France.

*filip@ optics.upol.cz

julien.laurat@sorbonne-universite.fr

[1] W. H. Zurek, Decoherence, Einselection, and the quantum origins of the classical, Rev. Mod. Phys. 75, 715 (2003).

[2] S. Haroche and J.-M. Raimond, Exploring the Quantum: Atoms, Cavities and Photons (Oxford University Press, Oxford, 2006).

[3] C. Monroe, D. M. Meekhof, B. E. King, and D. J. Wineland, A Schrödinger cat superposition state of an atom, Science 272, 1131 (1996).

[4] C. J. Myatt, B. E. King, Q. A. Turchette, C. A. Sackett, D. Kielpinski, W. M. Itano, C. Monroe, and D. J. Wineland, Decoherence of quantum superpositions through coupling to engineered reservoirs, Nature (London) 403, 269 (2000).
[5] M. J. McDonnell, J. P. Home, D. M. Lucas, G. Imreh, B. C. Keitch, D. J. Szwer, N. R. Thomas, S. C. Webster, D. N. Stacey, and A. M. Steane, Long-Lived Mesoscopic Entanglement outside the Lamb-Dicke Regime, Phys. Rev. Lett. 98, 063603 (2007).

[6] H.-Y. Lo, D. Kienzler, L. de Clercq, M. Marinelli, V. Negnevitsky, B. C. Keitch, and J. P. Home, Spin motion entanglement and state diagnosis with squeezed oscillator wave packets, Nature (London) 521, 336 (2015).

[7] D. Kienzler, C. Flühmann, V. Negnevitsky, H.-Y. Lo, M. Marinelli, D. Nadlinger, and J. P. Home, Observation of Quantum Interference between Separated Mechanical Oscillator Wave Packets, Phys. Rev. Lett. 116, 140402 (2016).

[8] A. Ourjoumtsev, R. Tualle-Brouri, J. Laurat, and $\mathrm{Ph}$. Grangier, Generating optical Schrödinger kittens for quantum information processing, Science 312, 83 (2006).

[9] J. S. Neergaard-Nielsen, B. Melholt Nielsen, C. Hettich, K. Mølmer, and E. S. Polzik, Generation of a Superposition of Odd Photon Number States for Quantum Information Networks, Phys. Rev. Lett. 97, 083604 (2006).

[10] H. Takahashi, K. Wakui, S. Suzuki, M. Takeoka, K. Hayasaka, A. Furusawa, and M. Sasaki, Generation of Large-Amplitude Coherent-State Superposition via AncillaAssisted Photon Subtraction, Phys. Rev. Lett. 101, 233605 (2008).

[11] A. Ourjoumtsev, H. Jeong, R. Tualle-Brouri, and $\mathrm{Ph}$. Grangier, Generation of optical Schrödinger cats from photon number states, Nature (London) 448, 784 (2007).

[12] J. Etesse, M. Bouillard, B. Kanseri, and R. Tualle-Brouri, Experimental Generation of Squeezed Cat States with an Operation Allowing Iterative Growth, Phys. Rev. Lett. 114, 193602 (2015).

[13] K. Huang et al., Optical Synthesis of Large-Amplitude Squeezed Coherent-State Superpositions with Minimal Resources, Phys. Rev. Lett. 115, 023602 (2015).

[14] S. Deléglise, I. Dotsenko, C. Sayrin, J. Bernu, M. Brune, J.-M. Raimond, and S. Haroche, Reconstruction of nonclassical cavity field states with snapshots of their decoherence, Nature (London) 455, 510 (2008).

[15] B. Vlastakis, G. Kirchmair, Z. Leghtas, S. E. Nigg, L. Frunzio, S. M. Girvin, M. Mirrahimi, M. H. Devoret, and R. J. Schoelkopf, Deterministically encoding quantum information using 100-Photon Schrödinger cat states, Science 342, 607 (2013).

[16] M. Brune, E. Hagley, J. Dreyer, X. Maître, A. Maali, C. Wunderlich, J. M. Raimond, and S. Haroche, Observing the Progressive Decoherence of the "Meter" in a Quantum Measurement, Phys. Rev. Lett. 77, 4887 (1996).

[17] P. W. Shor, Scheme for reducing decoherence in quantum computer memory, Phys. Rev. A 52, R2493 (1995).

[18] A. Steane, Error Correcting Codes in Quantum Theory, Phys. Rev. Lett. 77, 793 (1996).

[19] M. Mirrahimi, Z. Leghtas, V. V. Albert, S. Touzard, R. J. Schoelkopf, L. Jiang, and M. H. Devoret, Dynamically protected cat-qubits: a new paradigm for universal quantum computation, New J. Phys. 16, 045014 (2014).

[20] C. H. Bennett, D. P. DiVincenzo, J. A. Smolin, and W. K. Wootters, Mixed-state entanglement and quantum error correction, Phys. Rev. A 54, 3824 (1996). 
[21] D. Vitali, P. Tombesi, and G. J. Milburn, Controlling the Decoherence of a Meter, via Stroboscopic Feedback, Phys. Rev. Lett. 79, 2442 (1997).

[22] C. Sayrin et al., Real-time quantum feedback prepares and stabilizes photon number states, Nature (London) 477, 73 (2011).

[23] T. A. B. Kennedy and D. F. Walls, Squeezed quantum fluctuations and macroscopic quantum coherence, Phys. Rev. A 37, 152 (1988).

[24] A. Serafini, S. De Siena, F. Illuminati, and M. G. A. Paris, Minimum decoherence cat-like states in Gaussian noisy channels, J. Opt. B 6, S591 (2004).

[25] R. Filip, Amplification of Schrödinger-cat state in a degenerate optical parametric amplifier, J. Opt. B 3, S1 (2001).

[26] R. Filip, Gaussian quantum adaptation of non-Gaussian states for a lossy channel, Phys. Rev. A 87, 042308 (2013).

[27] W. P. Schleich, Quantum Optics in Phase Space (Wiley, Berlin, 2001).

[28] D. Menzies and R. Filip, Gaussian-optimized preparation of non-Gaussian pure states, Phys. Rev. A 79, 012313 (2009).

[29] Y. Miwa, J. I. Yoshikawa, N. Iwata, M. Endo, P. Marek, R. Filip, P. van Loock, and A. Furusawa, Exploring a New Regime for Processing Optical Qubits: Squeezing and Unsqueezing Single Photons, Phys. Rev. Lett. 113, 013601 (2014).

[30] See Supplemental Material at http://link.aps.org/ supplemental/10.1103/PhysRevLett.120.073603 for description of the experimental setup and theoretical derivation of rate of decay for squeezed and optimally-squeezed CSS.

[31] J. P. Paz, S. Habib, and W. H. Zurek, Reduction of the wave packet: Preferred observable and decoherence time scale, Phys. Rev. D 47, 488 (1993).

[32] O. Morin, V. D’Auria, C. Fabre, and J. Laurat, High-fidelity single-photon source based on a type II optical parametric oscillator, Opt. Lett. 37, 3738 (2012).

[33] H. Le Jeannic, V. B. Verma, A. Cavaillès, F. Marsili, M. D. Shaw, K. Huang, O. Morin, S. W. Nam, and J. Laurat, Highefficiency superconducting nanowire single-photon detector for quantum state engineering in the near infrared, Opt. Lett. 41, 5341 (2016).

[34] K. Huang, H. Le Jeannic, V. B. Verma, M. D. Shaw, F. Marsili, S. W. Nam, E. Wu, H. Zeng, O. Morin, and J. Laurat, Experimental quantum state engineering with timeseparated heraldings from a continuous-wave light source: A temporal-mode analysis, Phys. Rev. A 93, 013838 (2016).

[35] A. I. Lvovsky and M. G. Raymer, Continuous-variable optical quantum-state tomography, Rev. Mod. Phys. 81, 299 (2009).

[36] O. Morin, C. Fabre, and J. Laurat, Experimentally Accessing the Temporal Mode of Traveling Quantum Light States, Phys. Rev. Lett. 111, 213602 (2013).
[37] M. Ho, O. Morin, J.-D. Bancal, N. Gisin, N. Sangouard, and J. Laurat, Witnessing single-photon entanglement with local homodyne measurements: Analytical bounds and robustness to losses, New J. Phys. 16, 103035 (2013).

[38] Z. Leghtas, G. Kirchmair, B. Vlastakis, R. J. Schoelkopf, M. H. Devoret, and M. Mirrahimi, Hardware-Efficient Autonomous Quantum Memory Protection, Phys. Rev. Lett. 111, 120501 (2013).

[39] M. Bergmann and P. van Loock, Quantum error correction against photon loss using multicomponent cat states, Phys. Rev. A 94, 042332 (2016).

[40] R. W. Heeres, Ph. Reinhold, N. Ofek, L. Frunzio, L. Jiang, M. H. Devoret, and R. J. Schoelkopf, Implementing a universal gate set on a logical qubit encoded in an oscillator, Nat. Commun. 8, 94 (2017).

[41] E. Flurin, V. V. Ramasesh, S. Hacohen-Gourgy, L. S. Martin, N. Y. Yao, and I. Siddiqi, Observing Topological Invariants Using Quantum Walks in Superconducting Circuits, Phys. Rev. X 7, 031023 (2017).

[42] P. van Loock, T. D. Ladd, K. Sanaka, F. Yamaguchi, K. Nemoto, W. J. Munro, and Y. Yamamoto, Hybrid Quantum Repeater Using Bright Coherent Light, Phys. Rev. Lett. 96, 240501 (2006).

[43] F. Toscano, D. A. R. Dalvit, L. Davidovich, and W. H. Zurek, Sub-Planck phase-space structures and Heisenberg-limited measurements, Phys. Rev. A 73, 023803 (2006).

[44] M. Yukawa, K. Miyata, T. Mizuta, H. Yonezawa, P. Marek, R. Filip, and A. Furusawa, Generating superposition of up-to three photons for continuous variable quantum information processing, Opt. Express 21, 5529 (2013).

[45] M. Hofheinz et al., Synthesizing arbitrary quantum states in a superconducting resonator, Nature (London) 459, 546 (2009).

[46] O. Morin, K. Huang, J. Liu, H. Le Jeannic, C. Fabre, and J. Laurat, Remote creation of hybrid entanglement between particle-like and wave-like optical qubits, Nat. Photonics $\mathbf{8}$, 570 (2014).

[47] H. Jeong, A. Zavatta, M. Kang, S.-W. Lee, L. S. Costanzo, S. Grandi, T. C. Ralph, and M. Bellini, Generation of hybrid entanglement of light, Nat. Photonics 8, 564 (2014).

[48] J. Fiurášek, Efficient representation of purity-preserving Gaussian quantum filters, Phys. Rev. A 87, 052301 (2013).

[49] B. Hage, A. Samblowski, J. DiGuglielmo, A. Franzen, J. Fiurášek, and R. Schnabel, Preparation of distilled and purified continuous-variable entangled states, Nat. Phys. 4, 915 (2008).

[50] U. L. Andersen, J. S. Neergaard-Nielsen, P. van Loock, and A. Furusawa, Hybrid discrete- and continuous-variable quantum information, Nat. Phys. 11, 713 (2015). 